\title{
Machine Learning Will Change Medicine
}

\author{
Michael Forsting \\ Essen University Hospital, University of Essen-Duisburg, Essen, Germany
}

$\mathbf{W}$

hy do we need artificial intelligence (AI) algorithms, or machine learning, in medicine? Is there not enough "natural" intelligence among physicians? Well, that clearly depends on the individual medical education of every single physician. But to be honest, no doctor in the world can be perfectly up-to-date on recent medical advances. We need help from the world of technology.

Besides the problem of data overflow, there are other reasons why we need AI in medicine. One main problem with making decisionsnot only in medicine but in general-is the optimism bias, or the difference between a person's expectation and the outcome that follows. In addition, physicians have an optimism bias of their own: they usually think that the patient's problem falls into the specific discipline to which the physician belongs. Imagine a patient with pain in his left arm. A cardiologist will always suppose first that this patient has a coronary problem, a neurosurgeon will check for a cervical disk problem, an orthopedic surgeon will have a look at the shoulder, and an angiologist will exclude a vascular problem. Someone will eventually figure out what the disease is, but it can take time. And now envision a system in which the patient first fills out a questionnaire based on numerous data obtained from patients with left-arm pain and their validated diagnoses. Such a system would clearly have no optimism bias. Imagine how helpful this tool could be in rural areas with few well-educated physicians. But even in high-end first-world countries, would we still need physicians to diagnose popular diseases or would a well-trained physician's assistant be enough?

We need AI for rare diseases. Some of the so-called rare diseases are probably not actually rare but just often overlooked. And those that really are rare are difficult to remember even for specialists. Think of the numerous syndromes. Intelligent systems never forget anything. Regardless of whether one is looking at images, laboratory values, or genetic data, well-trained intelligent systems will be faster and better than most physicians.

Will radiologists or nuclear medicine physicians be replaced by AI? Recently, Obermeyer and Emanuel stated that "machine learning will displace much of the work of radiologists" (1). It will change our daily life. It will remove a lot of the routine work. AI will make it pretty easy to diagnose simple things such as intracerebral bleeding, typical bone fractures, and liver cysts. Well-trained systems will become excellent advisers on mammograms, lung problems, and early-stroke CT interpretation. Scientists all over the world are

Received Jan. 21, 2017; revision accepted Jan. 24, 2017.

For correspondence or reprints contact: Institute of Diagnostic and Interventional Radiology and Neuroradiology, Essen University Hospital, University of Essen, Hufelandstrasse 55, D 45147 Essen, Germany.

E-mail: m.forsting@uni-essen.de

Published online Feb. 2, 2017.

COPYRIGHT (c) 2017 by the Society of Nuclear Medicine and Molecular Imaging. DOI: 10.2967/jnumed.117.190397 training systems to detect pulmonary embolism, characterize microcalcifications of the breast, and analyze the texture of the fetal brain and prostate cancer (2-5). Even the very first results are extremely promising.

Those who do not like that scenario usually come up with medicolegal issues. Who is responsible if the system fails-for example, if the system misdiagnoses? Well, if it could be said that no radiologist anywhere in the world ever fails, then failure of the system would be considered a major problem. Such is not the case. But besides that, look at laboratory medicine. Automatization in laboratory medicine has improved quality dramatically; we are not dependent any more on human skill in counting leukocytes, as we were 30 years ago. And because the question of who is responsible if the machine fails is clearly defined, there are strict quality control mechanisms in laboratory medicine. The same would become true for medical imaging: the system would see reference images all day, and the responsible physician would immediately realize when the system is out of order.

An important question will be who is in the driver's seat for the final decision. It is like the autopilot in an aircraft. If you ask who is flying the aircraft, the answer will be the pilot, the reason being that the human pilot is finally responsible for the action.

Initially, there will be at least two major problems in bringing AI to the medical imaging field. First, some diseases will not have a 1:1 translation into a specific image pattern. Information technology professionals may reply that radiologists probably do not see the specific pattern for a given disease right now, and this may be true for certain diseases. But from a pathophysiologic point of view, it is most likely that imaging will not always be specific. In these situations, the imaging specialist could be the integrator of imaging findings and other information about the patient. The system would provide a list of best matches from the imaging point of view, and the specialist would have to discuss the different possibilities with the referring physician.

The second major problem will be to define normal. The spine of a 20 -y-old patient looks different from that of a 60 -y-old, and among 60-y-old patients there is a huge variety of degeneration and often no correlation to back pain. Maybe it is not really helpful for a patient to learn that the degree of his spinal degeneration is beyond the 95th percentile for his age. Even if he never had back pain before, now he might get some!

Regardless, these problems will not prohibit AI from coming into the medical imaging arena. Imaging is pattern recognition, and computers can recognize patterns. What many people may not realize is that the deep-learning algorithms are there-in part, available as freeware. A couple of months ago, during a job interview with a young mathematician who works in a very active AI lab, I asked why AI is entering medicine so slowly. Her answer was simple: 
There are not enough medical datasets in the cloud to train our systems in medical problems.

If the main problem preventing $\mathrm{AI}$ from entering medicine (or imaging) is data-related, we have to reorganize our data. Imaging professionals all over the world host uncountable numbers of datasets. The problem with these data is that they are not the valid, annotated data required by learning systems. And that is true not only for imaging but for pathology, laboratory medicine, and-in the end-all fields of medicine. We need databases that can deliver these valid data to learning systems, and all the players in the health-care field will struggle for these datasets. The exciting question is who already owns or has these datasets, and who will earn money from them.

At this point, let's not look at problems with protecting data privacy, which can be solved quickly. Let's look at the datasets that are currently available - those of the device manufacturers, the first player in this field. The main problem with these data are that they will not be valid enough. They will be very heterogeneous. And then we will face the well-known information technology problem of garbage in, garbage out.

The imaging institutions themselves are clearly the second player. Perhaps huge institutions performing 100,000 or more CT scans per year will have departments for quality control that continuously feed learning systems with valid data. Perhaps we will have National Institutes of Health apps for lung diseases or Harvard apps for breast diseases. Or perhaps scientific societies-such as the Radiological Society of North American and the Society of Nuclear Medicine and Molecular Imaging — will identify AI as a new business area and create a structure allowing storage of valid data and training of AI systems.

Companies publishing medical textbooks or journals are the third player. They have a lot of valid data, and not only radiologic data. They can read textual data, and often even unstructured textual data.

I have been in radiology for 30 years now, and I do not believe that imaging will be in the driver's seat when it comes to the decision of who will organize the datasets. Device manufacturers will probably decide to deliver more than equipment. They may hire radiologists, create new departments for data quality and image annotation, and finally offer software-assisted reporting worldwide.

The evolution of laboratory medicine during the last 40 years is probably a good template for the development of all diagnostic disciplines, including medical imaging and nuclear medicine. This process of industrialization may not be as deep as in laboratory medicine because there will be no 1:1 correlation between every imaging study and a distinctive disease. And we will always have patients in our departments instead of probes. The patients will need an explanation of their images; they will need an injection of contrast agent and additional images or sequences. But there will be a substantial process of industrialization. And the need for diagnostic radiologists will probably decrease, as has the demand for physicians working in the field of laboratory medicine. I can imagine that there will be "diagnostic physicians" responsible for the integration of all diagnostic information-for example, "radiopathologists."

The fact is that-just as those who made horse-drawn buggies were not asked whether they liked the advent of automobiles-we as imaging physicians will not be asked whether we like this change or not. We can decide only whether we want to participate in its creation or ignore it.

In closing, some people hope that information technology in general, and maybe AI also, will reduce costs within the healthcare system. I am not convinced of that outcome. It was not digitalization of medical imaging that reduced its cost, it was the way we improved our services and our quality.

\section{DISCLOSURE}

No potential conflict of interest relevant to this article was reported.

\section{REFERENCES}

1. Obermeyer Z, Emanuel EJ. Predicting the future: big data, machine learning, and clinical medicine. $N$ Engl J Med. 2016;375:1216-1219.

2. Rucco M, Sousa-Rodrigues D, Merelli E, et al. Neural hypernetwork approach for pulmonary embolism diagnosis. BMC Res Notes. 2015;8:617.

3. Mohebian MR, Marateb HR, Mansourian M, Mañanas MA, Mokarian F. A hybrid computer-aided-diagnosis system for prediction of breast cancer recurrence (HPBCR) using optimized ensemble learning. Comput Struct Biotechnol J. 2016;15:75-85.

4. Levman J, Takahashi E. Multivariate analyses applied to healthy neurodevelopment in fetal, neonatal, and pediatric MRI. Front Neuroanat. 2016;9:163.

5. Rampun A, Tiddeman B, Zwiggelaar R, Malcolm P. Computer aided diagnosis of prostate cancer: a texton based approach. Med Phys. 2016;43:5412. 